\title{
Implantation of bioresorbable scaffolds under guidance of optical coherence tomography: Feasibility and pilot clinical results of a systematic protocol
}

\author{
Juan Luis Gutiérrez-Chico ${ }^{1,2}$, Carlos Cortés ${ }^{1,3}$, Michele Schincariol ${ }^{2}$, Ula Limon ${ }^{1}$, \\ Meric Yalcinli ${ }^{1}$, María-Alexandra Durán-Cortés ${ }^{1}$, Milosz Jaguszewski ${ }^{1,2}$ \\ ${ }^{1}$ Klinikum Frankfurt (Oder), Interventional Cardiology, Germany \\ ${ }^{2}$ Institute of Cardiovascular Translational Research of Atlantic (ICTRA), Berlin, Germany \\ ${ }^{3}$ Hospital Clínico Universitario de Valladolid, ICICOR, Spain
}

This paper was guest edited by Prof. Joanna Wykrzykowska

\begin{abstract}
Background: Herein is hypothesised that a comprehensive optical coherence tomography (OCT)-guided implantation protocol for bioresorbable scaffolds (BRS) can improve expansion and apposition, thus resulting in better clinical outcomes, particularly in reducing thrombotic events.

Methods: Patients considered suitable for BRS therapy in de novo coronary lesions underwent OCT. The predominant type of plaque was classified as lipidic, fibrous or calcific. Accordingly they underwent tailored plaque preparation. After proper sizing, BRS was deployed and final OCT was acquired. Post-dilation was performed only in cases of suboptimal deployment. Procedural and 12 month clinical follow-up is reported.

Results: Twenty nine patients (41 lesions) who were considered clinically and angiographically suitable for BRS were enrolled, including challenging clinical scenarios such as ST-segment elevation myocardial infarction or CTOs. The OCT-guided protocol was feasible in $90.2 \%$ of the lesions: $14(37.8 \%)$ lipidic, 11 (29.7\%) fibrous, and 12 (32.4\%) calcific. Three (8\%) lesions classified as calcific were changed to treatment with metallic stent. BRS were implanted in 34 (91.9\%) lesions, thereof $30(88.2 \%)$ with optimal deployment in OCT. One (3.6\%) periprocedural MI occurred, resulting in $3.6 \%$ target vessel failure and 0\% scaffold thrombosis of any kind after a 12 month follow-up.

Conclusions: OCT-guided BRS implantation is feasible in $90.2 \%$ of de novo lesions and results in optimal expansion and apposition, correlating with $3.6 \%$ incidence of target vessel failure and $0 \%$ scaffold thrombosis at $12 \mathrm{~m}$ follow-up, probably due to better selection of lesions amenable for BRS treatment and to a possibility of tailoring intervention to the type of plaque. These encouraging pilot results require confirmation in larger clinical studies. (Cardiol J 2018; 25, 4: 443-458)
\end{abstract}

Key words: tomography, optical coherence, bioresorbable scaffold, percutaneous coronary intervention

\section{Introduction}

The advent of bioresorbable scaffolds (BRS) has been heralded as the fourth revolution in interventional cardiology, culminating three preced- ing cornerstones: balloon percutaneous coronary intervention $(\mathrm{PCI})$, bare-metal coronary stents (BMS) and drug-eluting stents (DES) [1]. The concept of transiently scaffolding the coronary artery during the minimal period after PCI, which

Address for correspondence: Prof. Juan Luis Gutiérrez-Chico, MD, PhD, FESC, FACC, Head of interventional Cardiology, Klinikum Frankfurt (Oder), Müllroser Chaussée 7, 15236 - Frankfurt (Oder), Germany, tel: +49 (0) 17630585019 , +34 615 319370, e-mail: juanluis.gutierrezchico@ictra.es

Received: 12.12.2017 Accepted: 10.05.2018 
is required to avoid acute closure, recoil and negative remodeling was of the utmost appeal [2]. The bioresorption ultimately results in the complete removal of all foreign material from the vessel wall, thus restoring vasomotion and normal physiology, avoiding the jailing of side branches, as well as reducing chronic inflammatory stimulus and mechanical stress on the vessel. This was theoretically expected to translate into eradication of very-late device thrombosis, and a substantial reduction of restenosis, neoatherosclerosis and late catch-up.

Nonetheless, all these foreseen theoretical advantages are failing to be empirically demonstrated in a clinical scenario hitherto. After initially encouraging results [3-9], BRS resulted in much higher device thrombosis rates than DES [10-13], most of them occurring acute or subacutely [10], but remained high in late and very late phases [11-14]. Furthermore, the incidence of periprocedural myocardial infarction (MI) and occlusion of small side branches were both higher in BRS than DES, due to the larger size of their struts [15], and cases of neoatherosclerosis have also been reported [16, 17]. The reasons for this mismatch between obvious theoretical advantages of BRS and their poorer-than-expected clinical performance remains unclear. The role of incomplete scaffold apposition (ISA) has been perhaps overemphasised, even though evidence linking acute ISA with device thrombosis is indirect and weak [18-20]. Rupture of the scaffold structure [21, 22] or underexpansion of the device [11,23-25] might likely explain cases of device failure. Indeed in most cases of scaffold thrombosis a mechanical cause can be identified in optical coherence tomography (OCT) [26].

Bare-metal coronary stents have very distinct physical properties from metallic DES: they are more fragile and prone to rupture, so deployment must be performed carefully, paying special attention to calcium [21, 27, 28]. In addition, they have limited overexpansion capacity [27-29], so an accurate sizing is crucial. Therefore BRS cannot be implanted as a conventional metallic stent: they require a BRS-dedicated implantation protocol, which takes into account their specific physical properties and peculiarities. The clinical outcome after BRS implantation improves sensibly with systematic use of quantitative coronary arteriography for accurate sizing [30] or after implementation of BRS-dedicated implantation protocols [11]. When the first approved BRS obtained the CE-mark in 2011, the manufacturer and experts recommended an angiography-guided implantation protocol [30], notwithstanding the limitations of angiography to assess critical features for accurate deployment, such as plaque composition, expansion, scaffold rupture or even for precise sizing if confounding elements (like calcium, foreshortening or vessel overlap) are present. At that time OCT was not as widespread as it is nowadays and it was considered rather a research tool without clear clinical applications. Nonetheless OCT is progressively generating an interesting corpus of evidence and already has clinical indications endorsed by the guidelines [31]. As a consequence, OCT is amply available in academic and non-academic centres now, so an OCT-guided implantation protocol for BRS would be currently feasible and could be adopted by a majority of implanting centres.

It is hypothesised herein that OCT-guided implantation protocol for BRS can accurately assess all anatomical variables that can interfere with optimal deployment, thus enabling a finely tailored BRS implantation that may translate into improved clinical outcomes. The goal of the current pilot study is to test the feasibility and safety of a protocol proposal to be used in larger, future studies to test this hypothesis.

\section{Methods}

This is a descriptive single-center pilot study to test the feasibility and safety of an OCT-guided implantation protocol for BRS, taking into account the physical properties of the device, the current evidence as well as the experience of the investigators.

\section{Study population}

Consecutive patients with an indication for PCI in the Hospital of Frankfurt Oder (Germany) and with coronary lesions considered angiographically suitable for BRS implantation were included in this study. Exclusion criteria were: 1 ) age $>80$ years old; 2) creatine clearance $<30 \mathrm{~mL} / \mathrm{min}$; 3 ) hemodynamic instability; 4) active bleeding; 5) any condition conferring a life expectancy $<10$ years; 6) excessive tortuosity; 7) severe calcification in the angiography; 8) target lesion involving the left main; 9) in-stent restenosis; 10) ostial lesion; 11) target lesion in a by-pass graft; 12) reference vessel diameter $<2.25 \mathrm{~mm}$ or $>4 \mathrm{~mm}$; 13) operator not certified for BRS implantation; 14) operator unable to acquire quality OCT or to properly interpret it; 15) mismatch between the lesion size and the size of the available BRS devices. 


\begin{tabular}{|c|c|c|c|}
\hline & ABSORB & DESOLVE & MAGMARIS \\
\hline Company & Abbott & Elixir & Biotronik \\
\hline Polymer & PLLA & PLLA-based & Magnesium \\
\hline Hoops & $\begin{array}{l}\text { In-phase } \\
\text { sinusoidal }\end{array}$ & In-phase sinusoidal & In-phase sinusoidal \\
\hline Peaks per hoop & 6 & 9 & 6 \\
\hline Connectors per hoop & 3 , peak-valley & 3 , peak-valley & 2, slopes \\
\hline Strut thickness $(\mu \mathrm{m})$ & 157 & $150-100$ & 150 \\
\hline \multicolumn{4}{|l|}{ Strut width $(\mu \mathrm{m})$} \\
\hline Hoop & 191 & 165 & 150 \\
\hline Connector & 140 & 100 & $80-100$ \\
\hline Vessel coverage $(\%)$ & 27 & 30 & 30 \\
\hline Potential cell diameter $(\mathrm{mm})$ & 3.0 & 3.2 & 3.0 \\
\hline Drug & Everolimus & Novolimus & Sirolimus \\
\hline Deployment & Slow & Slow & Quick \\
\hline OCT & Translucent & Translucent (sparkling) & Shadow \\
\hline
\end{tabular}

Figure 1. Composition, design and technical specification of bioresorbable scaffolds included in the study; OCT — optical coherence tomography; PLLA — poly-L-lactic acid.

The study was conducted according to the Declaration of Helsinki. Since all the steps in the hereby described intervention conform to currently approved standards of good clinical practice and only data stemming from a single centre were analyzed, the approval of the Ethics Committee could be waived and no specific informed consent was provided to the patients, other than the ones conventionally used for PCI with BRS and for the use of invasive imaging.

\section{Study devices}

The protocol applies the three BRS currently available in Europe: two polylactide-based BRS (ABSORB, Abbott Vascular, Santa Clara, CA, USA; DESOLVE, Elixir Medical Corp., Sunnyvale, CA, USA) and one magnesium BRS (MAGMARIS, Biotronik AG, Bülach, $\mathrm{CH}$ ).

The ABSORB (Abbott Vascular, Santa Clara, CA, USA) consists of a semi-crystalline poly(L-lactide) backbone and conformal coating of amorphous poly (D,L-lactide) that carries the antiproliferative drug everolimus. The scaffold is designed as inphase sinusoidal hoops, with 6 peaks and valleys per hoop and 3 straight longitudinal connectors linking peak-to-peak of adjacent hoops [32]. The strut thickness in radial direction is $158 \mu \mathrm{m}$ [33], while the width in the circumferential direction is $191 \mu \mathrm{m}$ for the hoop and $140 \mu \mathrm{m}$ for the connector (Fig. 1) [27]. The molecular weight of polymers is degraded primarily through hydrolysis of the ester bonds present in each monomer subunit. Crystalline residues $>2 \mu \mathrm{m}$ are phagocytosed by macrophages. Ultimately, the poly-lactide polymers degrade to lactate, which is metabolised via Krebs' cycle and other metabolic pathways, resulting in $\mathrm{CO}_{2}$ and $\mathrm{H}_{2} \mathrm{O}$ [34].

The DESOLVE (Elixir Medical Corp., Sunnyvale, CA, USA) is a poly-lactide-based BRS, eluting the antiproliferative drug novolimus. The scaffold is also designed as in-phase sinusoidal hoops, with a variable number of peaks and valleys per hoop, depending on the calibre of the device (9 peaks and valleys in the $3 \mathrm{~mm}$ DESOLVE) and also 3 straight longitudinal connectors linking peak-to-peak of adjacent hoops. The most proximal and most distal hoops are however out-of-phase, so peaks and valleys connect directly. The strut thickness in radial direction is $150 \mu \mathrm{m}$, while the width in circumferential direction is $165 \mu \mathrm{m}$ for the hoops and $100 \mu \mathrm{m}$ for the connector (Fig. 1) [28]. 


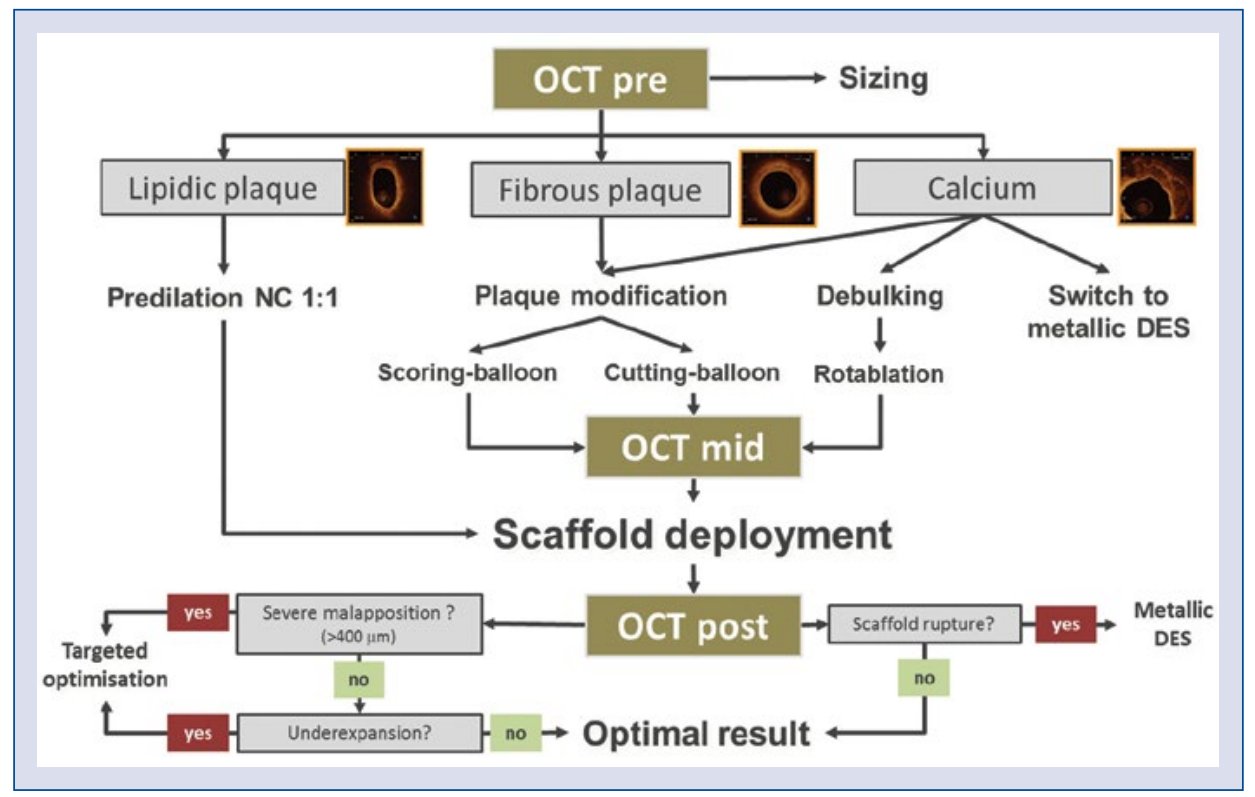

Figure 2. Optical coherence tomography (OCT)-guided bioresorbable scaffold implantation protocol; DES — drug-eluting stent; NC - non-compliant balloon.

The MAGMARIS (Biotronik AG, Bülach, CH) consists of a proprietary magnesium alloy and conformal coating of amorphous poly(L-lactide) that carries the antiproliferative drug sirolimus. The scaffold is designed as in-phase sinusoidal hoops, with 6 peaks and valleys per hoop and 2 sinusoidal longitudinal connectors linking the slopes between peaks and valleys of adjacent hoops [35]. The strut thickness in radial direction is $150 \mu \mathrm{m}$, while the width in circumferential direction is $140 \mu \mathrm{m}$ for the hoops and $80-100 \mu \mathrm{m}$ for the connector (Fig. 1). The magnesium $(\mathrm{Mg})$ resorption takes place in two steps: firstly, the Mg alloy reacts with water to create Mg-hydroxide; secondly, the Mg-hydroxide is slowly converted to amorphous calcium phosphate with high water content [36]. There is scarce bench-testing data from magnesium BRS (MgBRS) as from polylactide-based BRS [35]. Until more detailed information is available, it is reasonable to assimilate the MgBRS to polylactide BRS and hence following a cautionary imaging-guided implantation protocol.

\section{OCT-guided BRS implantation protocol}

PCI preparation and OCT acquisition. The PCI was performed via radial or femoral access with $\mathrm{a} \geq 6 \mathrm{~F}$ guiding catheter. A conventional 0.014" PCI wire as per operator choice was advanced distally to the target lesion. In case of ST-elevation MI with thrombolysis in myocardial infarction (TIMI) 0 flow or angiographic image of thrombus [37], aspiration thrombectomy was encouraged before OCT acquisition. OCT pre-intervention was acquired with a Dragonfly catheter and an Ilumien Optis console (St. Jude Medical, St. Paul, MN, USA) at a rotation speed of $180 \mathrm{~Hz}$ and a pullback speed of $18 \mathrm{~mm} / \mathrm{s}$, according to a non-occlusive technique [38]. Other pullback speeds were allowed if the operator justified the decision due to need of sparing contrast in patients with advanced renal insufficiency, long lesions or requiring multiple pullbacks.

OCT pre-intervention: the critical step. The OCT-pre was analyzed by an operator online with two specific aims: to select an accurate sizing and to evaluate histological type of plaque (Fig. 2).

Proximal and distal landing zones were selected, as defined by the closest plaque-free crosssections to the point of minimal lumen area (MLA) in the proximal and distal reference segments, respectively. If these definitions could not be met, the operator could select the landing zones by approximation (least diseased cross-section with functionally non-obstructive lumen area) or based in other interventional criteria, like preservation of side-branches. The length of the scaffoldingrequiring segment (SRS) was measured between the cross-sections of the proximal and distal landing zones. The proper size of the BRS was then selected, so the diameter did not differ by more than $0.5 \mathrm{~mm}$ with any of the maximal lumen diameters in the landing zones and length exceeded was at least $2 \mathrm{~mm}$ proximally and distally to length of the 


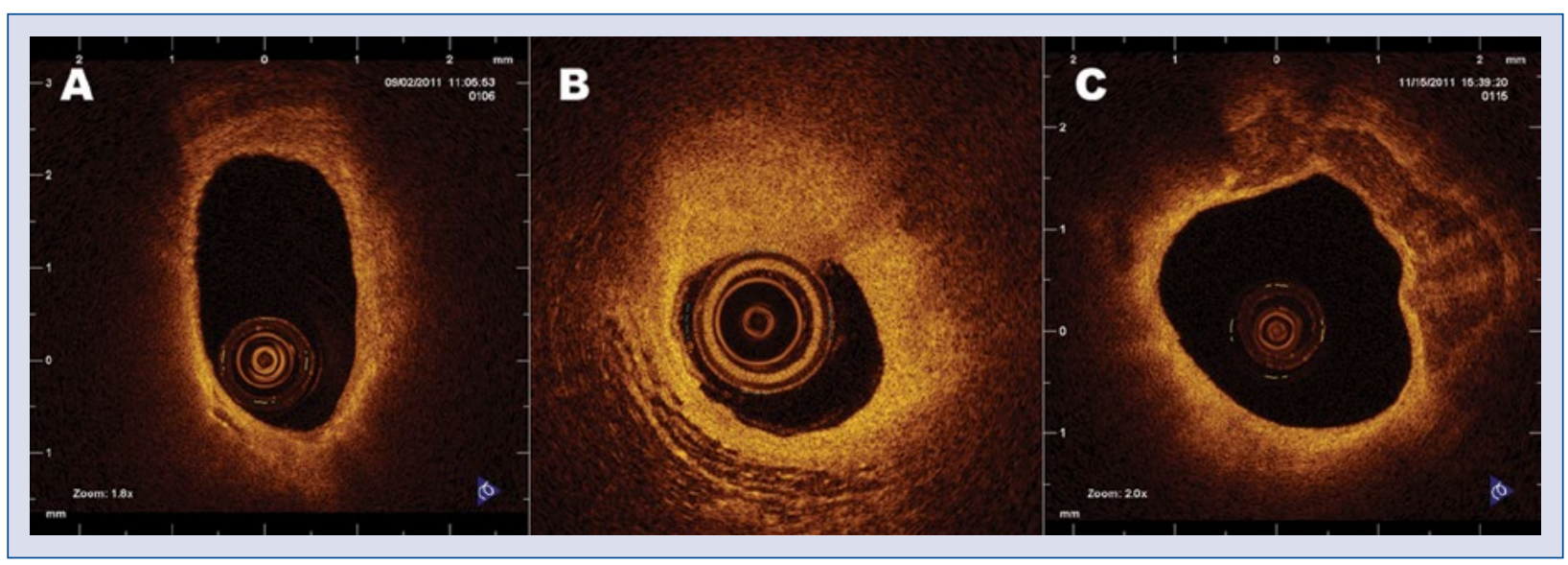

Figure 3. Type of plaque in optical coherence tomography. A. Lipidic plaque: low backscattering (hypointense) area very close to the lumen contour $(<300 \mu \mathrm{m})$ with very high attenuation (casting a shadow behind); B. Fibrotic plaque: high backscattering (hyperintense) throughout the plaque or at least in the most adluminal $300 \mu \mathrm{m}$, extending to a sector $\geq 135^{\circ}$; C. Calcific plaque: low backscattering (hypointense) area, at any depth between lumen and adventitia, but with low attenuation (no shadow behind), extending to a sector $\geq 135^{\circ}$.

SRS. In cases where the difference in diameter were $>0.5 \mathrm{~mm}$ in any of the landing zones, then an overlapping-BRS strategy should be considered, or the DESOLVE device (with accredited larger post dilation capabilities) was favoured. The length of BRS could be also adjusted according to interventional criteria, such as a wish to avoid BRS edge overhanging or jailing the take-off of side branches [39].

The type of plaque was assessed in the crosssection of MLA, which is automatically detected by current OCT software, and classified as lipidic, fibrous or calcific (Fig. 3). A lipidic plaque was defined as a low backscattering (hypointense) area very close to the lumen contour $(<300 \mu \mathrm{m})$ with very high attenuation (casting a shadow behind). A fibrous plaque was defined as tissue with high backscattering (hyperintense) throughout the plaque or at least in the most adluminal $300 \mu \mathrm{m}$, extending to a sector $\geq 135^{\circ}$. Finally, a calcific plaque was defined as a low backscattering (hypointense) area, at any depth between lumen and adventitia, but with low attenuation (no shadow behind), extending to a sector $\geq 135^{\circ}$. In cases of mixed patterns of plaque, or cross-sections meeting two or more definitions, the adjudication of type of plaque was done in a hierarchical manner, having priority given to calcific type, followed by fibrous and finally lipidic type. After having classified the type of plaque in MLA cross-section, the operator swept the proximal and distal segments of SRS. In cases where a different and higher hierarchy type of plaque were present in $>50 \%$ of any proximal or distal segments to MLA, then the plaque was hierarchically reclassified.

Plaque preparation and scaffold deployment. If the plaque was lipidic, then a 1:1 predilation with a non-compliant (NC) balloon was performed. If adequate expansion of the balloon was observed in the angiography, then the scaffold was deployed as previously described [11]. Fibrous plaques cannot be detected in angiography, but can prevent adequate expansion of BRS, even though sometimes the predilation balloon expands correctly in the angiography. Based on this observation, fibrous plaques ought to undergo plaque modification with scoring or cuttingballoons. If the plaque was calcific, the operator had to reconsider the suitability of the lesion for BRS and eventually be convinced to switch to a metallic DES. If the operator still preferred BRS, then aggressive plaque modification with scoringor cutting-balloons was mandatory. The plaque modification could be substituted by debulking with rotablation, if chosen by the operator in cases of calcific plaques (Fig. 2).

After plaque modification or debulking, the acquisition of another OCT pullback before scaffold deployment was compulsory in order to assess whether the modification had efficiently disrupted stricture areas and to check that no structural barrier remained in the artery that could prevent an adequate expansion. If these points could not be verified, the operator had to decide between performing further modification plus subsequent OCT iterations or switching to a metallic DES (Fig. 2). 
The sizing of the scaffold could be readjusted after this intermediate OCT pullback.

The scaffold was deployed following manufacturer recommendations: slow inflation (2 atmospheres every $2 \mathrm{~s}$ ) and keeping maximal pressure for $>30 \mathrm{~s}$ in polylactide BRS; whilst in MgBRS inflation could be performed at ordinary speed and keeping the maximal pressure for a prolonged interval was just optional.

OCT post-intervention. A repeat OCT pullback was acquired after scaffold deployment, following the same procedure as previously described, aiming at three specific parameters: expansion, apposition and rupture.

Expansion was quickly assessed online with the tool "lumen profile" of the Ilumen Optis software. The region of interest (ROI) was delimited by the scaffolded segment (most distal and most proximal cross-section in which struts were visible in all sectors of the circumference) and then MLA was automatically detected. If MLA was $<80 \%$ than both any proximal or any distal reference cross-sections within the ROI, the scaffold was considered underexpanded. Likewise, if severe ellipticity was found in any frame (ellipticity index $>1.6$ ), the scaffold was considered underexpanded as well, irrespective of its area. Ellipticity index was calculated by dividing the major diameter between the minor diameter of a cross section. In case of underexpansion, postdilation with an $\mathrm{NC}$ -balloon of the same size as the scaffold or $0.5 \mathrm{~mm}$ greater in calibre was mandatory.

Due to translucency of the polylactide polymers, ISA can be assessed directly in polylactide BRS [40]. Nonetheless Mg alloy is not translucent, so apposition in the MgBRS must be assessed as with metallic stents [40]. In order to propose a homogeneous criterion, valid for all BRS enrolled in this study, an ISA distance was chosen to be the distance between the adluminal edge of struts and lumen contour, in a line connecting the center of the adluminal strut edge with the center of gravity of the vessel, $>400 \mu \mathrm{m}$ was recommended threshold for optimization, since smaller ISA distances have proven to heal spontaneously in metallic DES [19]. In cases of an ISA distance $>400 \mu \mathrm{m}$, postdilation with a NC-balloon $0.5 \mathrm{~mm}$ larger than the nominal BRS calibre was recommended. Only if a DESOLVE BRS was implanted, in cases of severe ISA or postdilation with a NC-balloon $1.0 \mathrm{~mm}$ larger than the nominal BRS calibre was allowed.

Scaffold rupture was defined as the presence of stacked or overhanging struts in any crosssection [22]. Two-dimensional-cross-sectional im- ages were preferred for the assessment, because current OCT image-fusion (commonly dubbed 3D-OCT) is neither accurate nor reliable enough for this aim, particularly in BRS. Since scaffold rupture, even at subtle degrees, can be unstable and result in chronic repeat mechanical stress and incomplete vessel healing, cases of BRS rupture ought to be fixed and stabilised by implantation of a metallic DES.

\section{Objectives and clinical follow-up}

The main objectives of the study were the feasibility and safety of the OCT-guided BRS implantation protocol. Feasibility was defined as the proportion of interventions in which the protocol could be completed without violation in all interventions in which the protocol was planned. Safety was assessed as periprocedural complications and cumulated incidence of target vessel failure (cardiac death, vessel-related MI and target vessel revascularization) during the in-hospital period or at the $30^{\text {th }}$ day follow-up, whichever was longer. Secondary objectives were patient-oriented composite of all-cause death, MI or revascularization, together with individual components of composites, stent thrombosis, stroke, vascular complications, angiographic success (defined as $<20 \%$ diameter stenosis by visual estimation and TIMI 3 flow in the target vessel at the end of intervention) and procedural success (defined as angiographic success, plus absence of patient-oriented endpoint in the hospital phase or up to 30 day follow-up, whichever was longer), which conforms to Academic Research Consortium recommendations and definitions [41].

Telephone or visit follow-up were scheduled at 30 days, 6 months, 12 months and yearly for up to 5 years after the intervention. Due to the recent publication of discouraging clinical outcomes in BRS $[12,13]$ it was decided by the study investigators to communicate hereby at the interim 12 -month follow-up results to date, without waiting for the completion of the inclusion and follow-up, to meet the need of suggesting a possible future direction, potentially enabling improved clinical outcomes.

\section{Statistical analysis}

Continuous variables following Gaussian distribution were reported as mean \pm standard deviation, whilst they were reported as median (Q1-Q3) whenever a normal distribution could not be assumed. Nominal variables were reported as count (percent). Cumulated incidences are presented as proportions, calculated with respect to the total number of patients in whom OCT-guidance was 
feasible. All statistical analysis were performed using the IBM SPSS 20.0 (IBM Corp, Armonk, NY, USA) software package.

\section{Results}

Between January and July 2016, 29 patients (41 lesions) undergoing PCI in Hospital Frankfurt (Oder) in Germany were considered clinically and angiographically suitable for BRS implantation were included in the study. Tables 1 and 2 present clinical and angiographic characteristics of patients and lesions included, respectively.

In 1 patient (4 lesions) the OCT-pre could not be obtained due to excessive tortuosity of vessels. After analysis of OCT-pre, 14 (37.8\%) lesions were classified as lipidic, 11 (29.7\%) lesions as fibrous and $12(32.4 \%)$ lesions as calcific (Table 3, Fig. 4).

All lesions with lipidic plaque underwent unproblematic NC-balloon predilation and scaffold deployment. The OCT-post did not detect any cases of underexpansion, or BRS-rupture. Only $1(7.1 \%)$ case presented ISA $>400 \mu \mathrm{m}$ that could be corrected after targeted optimisation, so that all scaffolds (100) could be optimally deployed.

Eight $(72.7 \%)$ fibrotic lesions underwent plaque modification with scoring balloon (Angiosculpt, Biotronik AG, Bülach, $\mathrm{CH}$ ) and 3 (27.3\%) with cutting-balloon (Flextome, Boston Scientific, Marlborough, MA). In $2(18.2 \%)$ cases initially treated with scoring balloon, the OCT after plaque modification showed insufficient plaque disruption or persistence of structures jeopardising an optimal scaffold expansion, so a second dilation with scoring-balloon was required. In both cases, the second intermediate OCT showed sufficient plaque preparation and scaffolds could be deployed achieving optimal expansion, with no case of ISA requiring further optimization and no BRS rupture. Nonetheless, in the case requiring a second plaque modification with cutting-balloon, coronary rupture with pericardial tamponade occurred. The rupture was successfully handled with gentle compliant balloon inflation at the leak site (three consecutive inflations of $60 \mathrm{~s}$, with deflation pauses of $10 \mathrm{~s}$ in between) and pericardiocentesis. After restabilising the patient hemodynamically, the final OCT pullback showed optimal deployment of the scaffold and a completely sealed coronary artery. Cardiac markers raised over $3 \times$ ULN, but the patient had neither chest pain nor repolarisation changes in electrocardiogram. The patient could be uneventfully discharged 7 days after intervention (Fig. 5).
In 3 out of $12(25.0 \%)$ lesions with calcific plaque, the operator decided to switch to a metallic DES, due to massive calcification observed in OCT-pre, jeopardising an optimal scaffold deployment notwithstanding an aggressive and complex plaque preparation. Three (25.0\%) calcific lesions underwent plaque modification with scoring balloon and 5 (41.7\%) with cutting-balloon. Two of them required one further iteration, but both scaffolds were optimally deployed. In 2 cases the OCT inbetween was obviated due to the complexity of the procedure (CTO) and in both cases the final deployment was suboptimal due to underexpansion. One case met the definition of underexpansion due to ellipticity index $>1.7$, even though measured scaffold areas were quite uniform along the scaffolded segment. It corresponded to a chronic total occlusion in which proximal and distal true lumina had been connected through subintimal tracking. In the segment of subintimal scaffolding, the collapsed true lumen abutted into the neolumen created in the subintimal space, thus deforming the scaffold which was responsible for the high ellipticity index [42]. In this case, two NC-balloon inflations (with the same size of the scaffold and $0.5 \mathrm{~mm}$ bigger in diameter, respectively) left the ellipticity index exactly as it was at the beginning (Fig. 6). Finally, $1(8.3 \%)$ calcific plaque underwent rotablation with $1.5 \mathrm{~mm}$ burr, followed by successive plaque modification with a scoring balloon. After these manoeuvres scaffolds could be deployed successfully. ISA $>400 \mu \mathrm{m}$ was detected at the OCT post, but it could be corrected with post-dilation, thus achieving an optimal result.

According to the above reported data, the feasibility of OCT-guided protocol was $90.2 \%$ of initially enrolled lesions. BRS could finally be implanted in $34(91.9 \%)$ of the lesions undergoing OCT-guided protocol. 1 patient formally suffered periprocedural $\mathrm{MI}$ after prolonged intervention of a complex CTO (Table 4), asymptomatic and with normal systolic function in echocardiogram. At 12-month follow-up, 1 patient had died due to urosepsis and another patient underwent PCI due to non-ST-segment elevation MI in a non-target vessel (Table 4). These data correspond to an angiographic success of $100 \%$ and a procedural success of $96.4 \%$. There was no incidence of definite, probable or possible stent thrombosis.

\section{Discussion}

The main findings of this study can be summarised as follows: 1) OCT-guided BRS implantation 
Table 1. Clinical characteristics of the patients.

\begin{tabular}{|c|c|c|}
\hline & Initially enrolled ( $n=29$ ) & Finally treated with BRS $(n=25)$ \\
\hline Male & $22(75.9 \%)$ & $18(72.0 \%)$ \\
\hline Age [years] & $69.12(60.09-69.11)$ & 70.18 (62.53-77.85) \\
\hline Body mass index & 28.09 (25.06-31.37) & $28.09(24.50-31.37)$ \\
\hline \multicolumn{3}{|l|}{ Cardiovascular risk factors: } \\
\hline Hypertension & $24(82.8 \%)$ & $21(84.0 \%)$ \\
\hline Hypercholesterolemia & $11(37.9 \%)$ & $9(36.0 \%)$ \\
\hline \multicolumn{3}{|l|}{ Diabetes mellitus: } \\
\hline Type 2 on OAD & $13(44.8 \%)$ & $12(48.0 \%)$ \\
\hline Type 2 insulin-requiring & $1(3.4 \%)$ & $1(4.0 \%)$ \\
\hline \multicolumn{3}{|l|}{ Smoking: } \\
\hline Non smoker & $21(72.4 \%)$ & $19(76.0 \%)$ \\
\hline Previous smoker & $2(6.9 \%)$ & $1(4.0 \%)$ \\
\hline Current smoker & $6(20.7 \%)$ & $5(20.0 \%)$ \\
\hline Family history of CHD & $0(0.0 \%)$ & $0(0.0 \%)$ \\
\hline Previous MI & $6(20.7 \%)$ & $5(20.0 \%)$ \\
\hline \multicolumn{3}{|l|}{ Previous revascularization: } \\
\hline $\mathrm{PCl}$ & $12(41.4 \%)$ & $11(44.0 \%)$ \\
\hline CABG & $1(3.4 \%)$ & $1(4.0 \%)$ \\
\hline GFR (Cockroft-Gault [mL/min]) & $90.44(56.56-124.36)$ & $80.23(51.28-124.22)$ \\
\hline Serum hemoglobin $[\mathrm{g} / \mathrm{dL}]$ & $13.27(11.95-14.61)$ & $13.37(12.08-14.84)$ \\
\hline LVEF [\%] & $65(45-71)$ & $65(45-71)$ \\
\hline \multicolumn{3}{|l|}{ Clinical indication: } \\
\hline Prognostic indication & $10(34.5 \%)$ & $9(36.0 \%)$ \\
\hline Stable angina & $12(41.4 \%)$ & $9(36.0 \%)$ \\
\hline Unstable angina & $2(6.9 \%)$ & $2(8.0 \%)$ \\
\hline NSTEMI & $4(13.8 \%)$ & $4(16.0 \%)$ \\
\hline STEMI & $1(3.4 \%)$ & $1(4.0 \%)$ \\
\hline \multicolumn{3}{|l|}{ Procedural variables: } \\
\hline LM disease & $3(10.3 \%)$ & $2(8.0 \%)$ \\
\hline LAD disease & $19(65.5 \%)$ & $16(64.0 \%)$ \\
\hline LCx disease & $20(69.0 \%)$ & $17(68.0 \%)$ \\
\hline $\mathrm{RCA}$ disease & $16(55.2 \%)$ & $14(56.0 \%)$ \\
\hline Syntax score & $9.00(5.00-20.75)$ & $9.00(4.50-24.00)$ \\
\hline Contrast volume $[\mathrm{mL}]$ & $230(159-300)$ & $238(170-300)$ \\
\hline Dose-area product $\left[\mathrm{Gy} \times \mathrm{cm}^{2}\right]$ & $152.63(58.46-180.97)$ & $152.63(57.46-179.02)$ \\
\hline Fluoroscopy time [min] & $18.00(11.48-22.40)$ & 17.07 (11.33-20.48) \\
\hline \multicolumn{3}{|l|}{ Medication at discharge: } \\
\hline ASA & $29(100 \%)$ & $25(100 \%)$ \\
\hline Clopidogrel & $22(75.9 \%)$ & $18(72.0 \%)$ \\
\hline Prasugrel & $4(13.8 \%)$ & $4(16.0 \%)$ \\
\hline Ticagrelor & $3(10.3 \%)$ & $3(12.0 \%)$ \\
\hline Warfarin & $1(3.4 \%)$ & $1(4.0 \%)$ \\
\hline Rivaroxaban & $0(0.0 \%)$ & $0(0.0 \%)$ \\
\hline Dabigatran & $1(3.4 \%)$ & $1(4.0 \%)$ \\
\hline
\end{tabular}

Data presented as number (percentage) or as median (Q1-Q3). ASA — acetylsalicylic acide; BRS — bioreorbable scaffolds; CABG — coronary artery bypass graft; CHD — coronary heart disease; GFR — glomerular filtration rate; LAD — left anterior descending; LCx - left circumflex; LM - left main; LVEF — left ventricular ejection fraction; MI - myocardial infarction; NSTEMI — non-ST-elevation myocardial infarction;

$\mathrm{OAD}$ — oral antidiabetics; PCI — percutaneous coronary intervention; RCA — right coronary artery; SB — side branch; STEMI — ST-segment elevation myocardial infarction 
Table 2. Angiographic characteristics of lesions and procedural details.

\begin{tabular}{|c|c|}
\hline Parameters & $\begin{array}{l}\text { Lesions } \\
(n=41)\end{array}$ \\
\hline \multicolumn{2}{|l|}{ Coronary segment (ACC) } \\
\hline Prox RCA & $4(9.8 \%)$ \\
\hline Mid RCA & $1(2.4 \%)$ \\
\hline Distal RCA/PD/PL & $3(7.3 \%)$ \\
\hline Prox LAD & $9(22.0 \%)$ \\
\hline Mid LAD & $6(14.6 \%)$ \\
\hline Dist LAD & $2(4.9 \%)$ \\
\hline Diagonal branches & $4(9.8 \%)$ \\
\hline Prox LCx & $4(9.8 \%)$ \\
\hline Intermediate/OM & $6(14.6 \%)$ \\
\hline Distal LCx & $2(4.9 \%)$ \\
\hline \multicolumn{2}{|l|}{ Lesion characteristics } \\
\hline \multicolumn{2}{|l|}{ Calcification: } \\
\hline None to little & $36(87.8 \%)$ \\
\hline Moderate to severe & $5(12.2 \%)$ \\
\hline \multicolumn{2}{|l|}{ Thrombus: } \\
\hline G0 - No thrombus & $39(95.1 \%)$ \\
\hline G1 - Possible thrombus & $1(2.4 \%)$ \\
\hline G5 - Total vessel occlusion & $1(2.4 \%)$ \\
\hline Bifurcation: & $14(34.1 \%)$ \\
\hline Medina 110 & $6(42.9 \%)$ \\
\hline Medina 100 & $2(14.3 \%)$ \\
\hline Medina 010 & $5(35.7 \%)$ \\
\hline Medina 001 & $1(7.1 \%)$ \\
\hline CTO & $3(7.3 \%)$ \\
\hline DS [\%] & $80.0(75.0-87.5)$ \\
\hline \multicolumn{2}{|l|}{ Initial TIMI flow: } \\
\hline TIMI 0 & $4(9.8 \%)$ \\
\hline TIMI 3 & $37(90.2 \%)$ \\
\hline \multicolumn{2}{|l|}{ Procedural variables } \\
\hline $\begin{array}{l}\text { Average number of scaffolds/ } \\
\text { /stents implanted }\end{array}$ & $1.0(1.0-1.5)$ \\
\hline $\begin{array}{l}\text { Total number of devices } \\
\text { implanted: }\end{array}$ & 51 \\
\hline BVS & $28(54.9 \%)$ \\
\hline Desolve & $13(25.5 \%)$ \\
\hline Magmaris & $4(7.8 \%)$ \\
\hline Resolute & $6(11.8 \%)$ \\
\hline Scaffold diameter & $3.00(2.50-3.00)$ \\
\hline Scaffold length & $23(18-28)$ \\
\hline Overlapping scaffolds & $12(29.3 \%)$ \\
\hline Postdilation & $16(39.0 \%)$ \\
\hline \multicolumn{2}{|l|}{ Angiographic result } \\
\hline Final DS [\%] & $0.0(0.0-0.0)$ \\
\hline Final TIMI flow 3 & $41(100 \%)$ \\
\hline Angiographic success & $41(100 \%)$ \\
\hline
\end{tabular}

Table 2 (cont.). Angiographic characteristics of lesions and procedural details.

\begin{tabular}{lc}
\hline Parameters & $\begin{array}{c}\text { Lesions } \\
\text { (n= } 41)\end{array}$ \\
\hline \multicolumn{2}{l}{ Procedural complications (lesion level) } \\
Coronary dissection & $5(12.2 \%)$ \\
Plaque shift & $1(2.4 \%)$ \\
SB occlusion & $1(2.4 \%)$ \\
Coronary rupture: & $2(4.9 \%)$ \\
Balloon occlusion & $2(100 \%)$ \\
Covered stent & $0(0.0 \%)$ \\
Pericardiocentesis & $1(50.0 \%)$ \\
Surgery & $0(0.0 \%)$ \\
Acute thrombosis & $1(2.4 \%)$ \\
Device dislodgement & $1(2.4 \%)$ \\
\hline
\end{tabular}

Data presented as total number, number (percentage) or as median (Q1-Q3). ACC - American College of Cardiology (classification of the coronary segments according to ACC); BVS - bioresorbable vascular scaffold; CTO - chronic total occlusion; DS - diameter stenosis; LAD - left anterior descending; LCx - left circumflex; $\mathrm{OM}$ - obtuse marginal; $\mathrm{PD}$ - posterior descending; $\mathrm{PL}$ - posterolateral; RCA — right coronary artery; SB — side branch; TIMI thrombolysis in myocardial infarction

protocol is feasible in $90.2 \%$ of de novo lesions, in a high complexity setting with minimal exclusion criteria; 2) OCT enables identification of three different types of plaque, with differing mechanical behaviours during BRS implantation, and to tailor intervention accordingly; 3) OCT allows for a most refined selection of patients for BRS implantation, excluding a subgroup of angiographically suitable patients in whom suboptimal deployment might be anticipated; 4) OCT-guided BRS implantation is safe and effective, resulting in optimal angiographic success, a minimal incidence of underexpansion or severe malapposition and completely avoiding scaffold rupture during the implantation. These optimal angiographic and imaging results correlate clinically with very low rates of periprocedural, inhospital and 12-month adverse events, preventing the occurrence of early or late scaffold thrombosis.

It has been sufficiently demonstrated that the high risk of scaffold thrombosis with BRS is partly due to an unwary implantation technique. Undisputable evidence for this causative association was empirically obtained in a Swiss-German registry of 1305 patients, in which the incidence of scaffold thrombosis was significantly reduced from $3.3 \%$ to $1.0 \%$ at 12 months after implementation of BRS-dedicated implantation protocol [11]. Still the incidence of scaffold thrombosis remained relatively high in subsequent clinical studies which had 
Table 3. Descriptive summary of the optical coherence tomography (OCT) findings and subsequent actions at different steps of the protocol.

\begin{tabular}{|c|c|c|}
\hline OCT guidance & Lesions ( $n=37$ ) & Reference \% \\
\hline OCT pre & $37(100 \%)$ & 37 (lesions) \\
\hline \multicolumn{3}{|l|}{ Sizing: } \\
\hline Proximal RVD [mm] & $2.99(2.57-3.37)$ & \\
\hline Distal RVD [mm] & $2.78(2.38-3.01)$ & \\
\hline Lesion length [mm] & $18(13-30)$ & \\
\hline \multicolumn{3}{|l|}{ Type of plaque: } \\
\hline Lipidic & $14(37.8 \%)$ & 37 (lesions) \\
\hline Fibrous: & $11(29.7 \%)$ & 37 (lesions) \\
\hline Scoring-balloon & $8(72.7 \%)$ & 11 (fibrous plaques) \\
\hline Cutting-balloon & $3(27.3 \%)$ & 11 (fibrous plaques) \\
\hline Calcific: & $12(32.4 \%)$ & 37 (lesions) \\
\hline Scoring-balloon & $3(25.0 \%)$ & 12 (calcific plaques) \\
\hline Cutting-balloon & $5(41.7 \%)$ & 12 (calcific plaques) \\
\hline Rotablation & $1(8.3 \%)$ & 12 (calcific plaques) \\
\hline Switch to DES & $3(25.0 \%)$ & 12 (calcific plaques) \\
\hline OCT in-between & $18(90.0 \%)$ & 20 (fibrous/calcific treated with BRS) \\
\hline Number of iterations & $1.00(1.00-1.25)$ & \\
\hline OCT post & $34(100 \%)$ & 34 (lesions treated with BRS) \\
\hline Underexpansion (area) & $4(11.8 \%)$ & 34 (lesions treated with BRS) \\
\hline Ellipticity index $\geq 1.6$ & $4(11.8 \%)$ & 34 (lesions treated with BRS) \\
\hline Underexpansion (area or ellipticity) & $5(14.7 \%)$ & 34 (lesions treated with BRS) \\
\hline ISA $>400 \mu \mathrm{m}$ & $5(14.7 \%)$ & 34 (lesions treated with BRS) \\
\hline Scaffold rupture & $0(0.0 \%)$ & 34 (lesions treated with BRS) \\
\hline Optimally implanted scaffold & $27(79.4 \%)$ & 34 (lesions treated with BRS) \\
\hline OCT post-optimisation & $8(23.5 \%)$ & 34 (lesions treated with BRS) \\
\hline Underexpansion (area) & $3(8.8 \%)$ & 34 (lesions treated with BRS) \\
\hline Ellipticity index $\geq 1.6$ & $4(11.8 \%)$ & 34 (lesions treated with BRS) \\
\hline Underexpansion (area or ellipticity) & $4(11.8 \%)$ & 34 (lesions treated with BRS) \\
\hline ISA $>400 \mu \mathrm{m}$ & $1(2.9 \%)$ & 34 (lesions treated with BRS) \\
\hline Optimally implanted scaffold & $30(88.2 \%)$ & 34 (lesions treated with BRS) \\
\hline
\end{tabular}

Data presented as number (percentage) or as median (Q1-Q3). BRS — bioresorbable scaffold; DES — drug-eluting stent; ISA — incomplete scaffold apposition; RVD - reference vessel diameter

already incorporated strict implantation protocols [13]. The causes for this persisting high incidence of thrombosis were most likely multifactorial, but could partly be explained because of the main large-scale BRS studies have followed angiography-guided implantation protocols that could not adjust to variable mechanical resistance of plaque and because of the use of invasive imaging, particularly OCT, which has been marginal [10-13]. The physical properties of BRS materials are very different from those of metallic stents: they are more fragile, prone to rupture under mechanical stress and have sensibly lower Young modulus for elastic deformation, so they cannot transmit the pressure of the balloon onto the atheroma plaque as efficiently as metallic stents. In other words, whilst the metal in DES is harder than any plaque component, the polymers in BRS are weaker than some of the plaque components they are supposed to crush and angiography is inherently limited to characterise them. The results of the present study confirm that current angiography-guided implantation is appropriate for lipidic plaques, in which $100 \%$ of the scaffolds were optimally deployed (93\% of them without postdilation) following similar recommendations to the current angiographic 


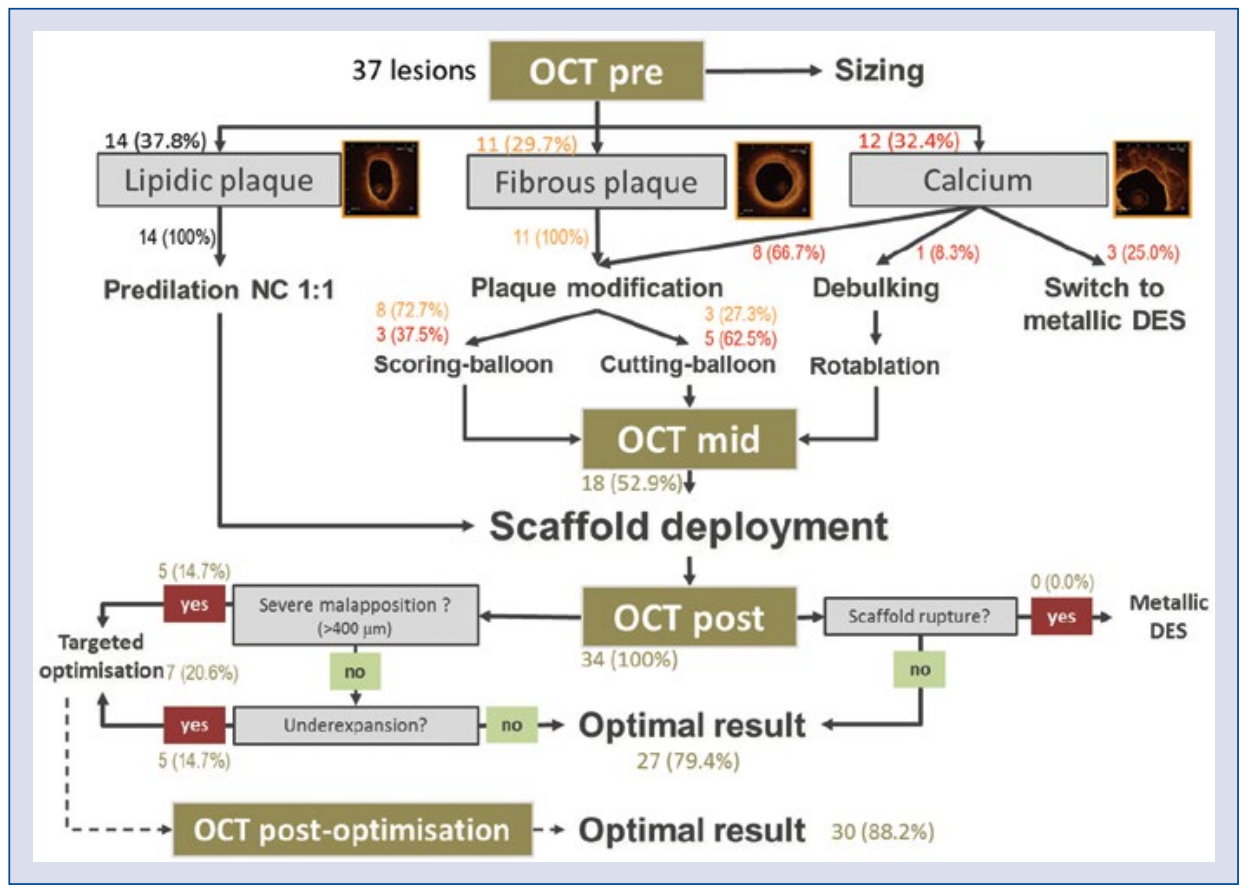

Figure 4. Distribution of types of lesions and different steps of the protocol; BRS - bioresorbable scaffold; DES — drug-eluting stent; NC — non-compliant balloon; OCT — optical coherence tomography.

standard [11], but lipidic plaques were only $38 \%$ in the present series. Conversely, angiography alone could be insufficient for fibrous or calcific plaques (62\% in present series), which involves all of the few cases of final suboptimal scaffold deployment. Calcium is amply recognised as a foe of BRS and can usually be detected in angiography by experienced operators, but angiography might lack the sensitivity to detect the level of calcification that disqualifies a lesion for BRS therapy as reflected by the fact that $8 \%$ of the lesions considered angiographically suitable for BRS were switched to DES after OCT examination. Moreover, neither angiography nor gray-scale intravascular ultrasound can reliably detect fibrous plaques that have been hitherto disregarded. A recent short case series has reported BRS underexpansion in fibrous plaques, even though the balloon expanded correctly in predilation. Considering these data altogether, the need for an OCT-guided implantation protocol in BRS seems justified until more compelling clinical evidence becomes available.

According to available research, this is the first comprehensive OCT-guided decision-tree for BRS implantation proposed and clinically tested hitherto. It is relevant to highlight that this protocol focuses on plaque preparation before scaffold deployment, rather than on postdilation. Young's modulus for elastic deformation under compres- sion in all BRS materials is much lower than in metallic DES, therefore once BRS has been deployed and interposed between the balloon and vessel wall, it might be too late for an attempt at optimization, especially regarding expansion. This has been the main reason to emphasise predilation instead of postdilation. The high proportion of procedural success and the minimal cumulative incidence of clinical events at 12-month follow-up might endorse this strategy as feasible and effective. OCT-guidance enables an accurate selection of patients and lesions in real-world conditions, as it permits tailoring intervention for each patient, escalating the aggressiveness of plaque preparation according to expected mechanical resistance of each plaque. Albeit this pilot series is modest to infer bold clinical conclusions, the results encourage further research in this direction. An important result worthy of attention is the $0 \%$ incidence of acute, subacute and late scaffold thrombosis in this series. In the first real-world registries of BRS up to $70 \%$ scaffold thrombosis was acute or subacute, thus strongly suggesting procedural factors as the most likely cause [10]. The prevention of thrombotic events by means of a strict imaging-guided implantation is in line with that interpretation and is reassuring of the role played by procedural factors in the risk of scaffold thrombosis. 


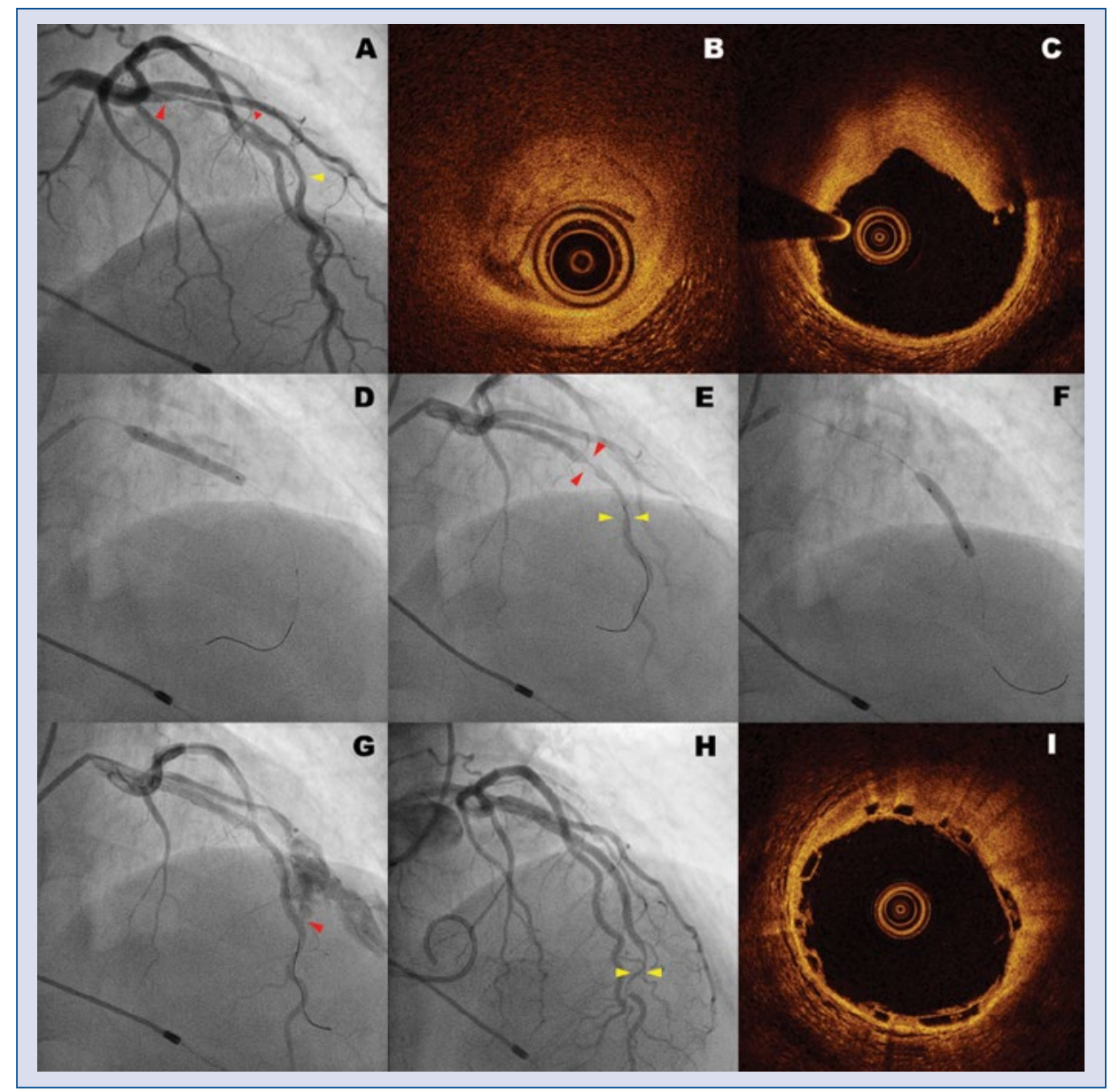

Figure 5. Case of coronary rupture and cardiac tamponade in patient with fibrotic lesion. A. Lesion in the proximal left anterior descending (LAD; red arrows), with plaque extending up to the mid-LAD (yellow arrow); B. Optical coherence tomography (OCT) pre was occlusive, but it shows a fibrotic plaque that was treated with cutting-balloon; C. OCT after cutting shows how the least diseased sectors of the cross-section (between 2 and 8 ) are also disrupted by the cutting balloon and appear now as a single layer corresponding to the adventitia, whilst the plaque fibrotic plaque is displaced into the opposite sector; D. ABSORB deployment after preparation of a fibrotic plaque with cutting-balIoon; E. Incomplete plaque coverage distally (red arrows), with plaque extending up to the mid-LAD (yellow arrow); F. Predilation with compliant balloon; G. Coronary rupture at the distal edge of the balloon predilation (red arrow), with blood extravasation into the pericardium; H. After pericardiocentesis and sealing of the rupture with balloon, a second ABSORB could be implanted distally, with good angiographic result; still some intracoronary haematoma (yellow arrows) can be distally noticed in the angiography; I. Optimal scaffold deployment in OCT. The patient underwent uneventful clinical outcome, notwithstanding the severe procedural complication.

The results of this study also highlights the limited role of postdilation in BRS, as anticipated. Postdilation has been dogmatically adopted as an undisputed requirement for optimal BRS implantation, but evidence for this recommendation is weak and debatable. In ABSORB Cohort B, some adverse events were attributed to aggressive postdilation [7], but posterior studies found high-pressure postdilation safe [43]. Nonetheless, quantitative analysis of the effect of postdilation in BRS suggested a negligible effect on expansion.
Summarizing, post-dilatation may not be harmful, but it seems to be ineffectual. Some evidence about the need of postdilation in BRS indeed, stems from systematic protocols that included postdilation but also aggressive predilation with NC balloons and BRS implantation deployed to an adequate balloon expansion [11]: systematic protocol improves clinical outcomes, but the effect of postdilation itself has never been explored. The present study reports a limited role for post-dilation: the proportion of optimally deployed scaffolds increased 


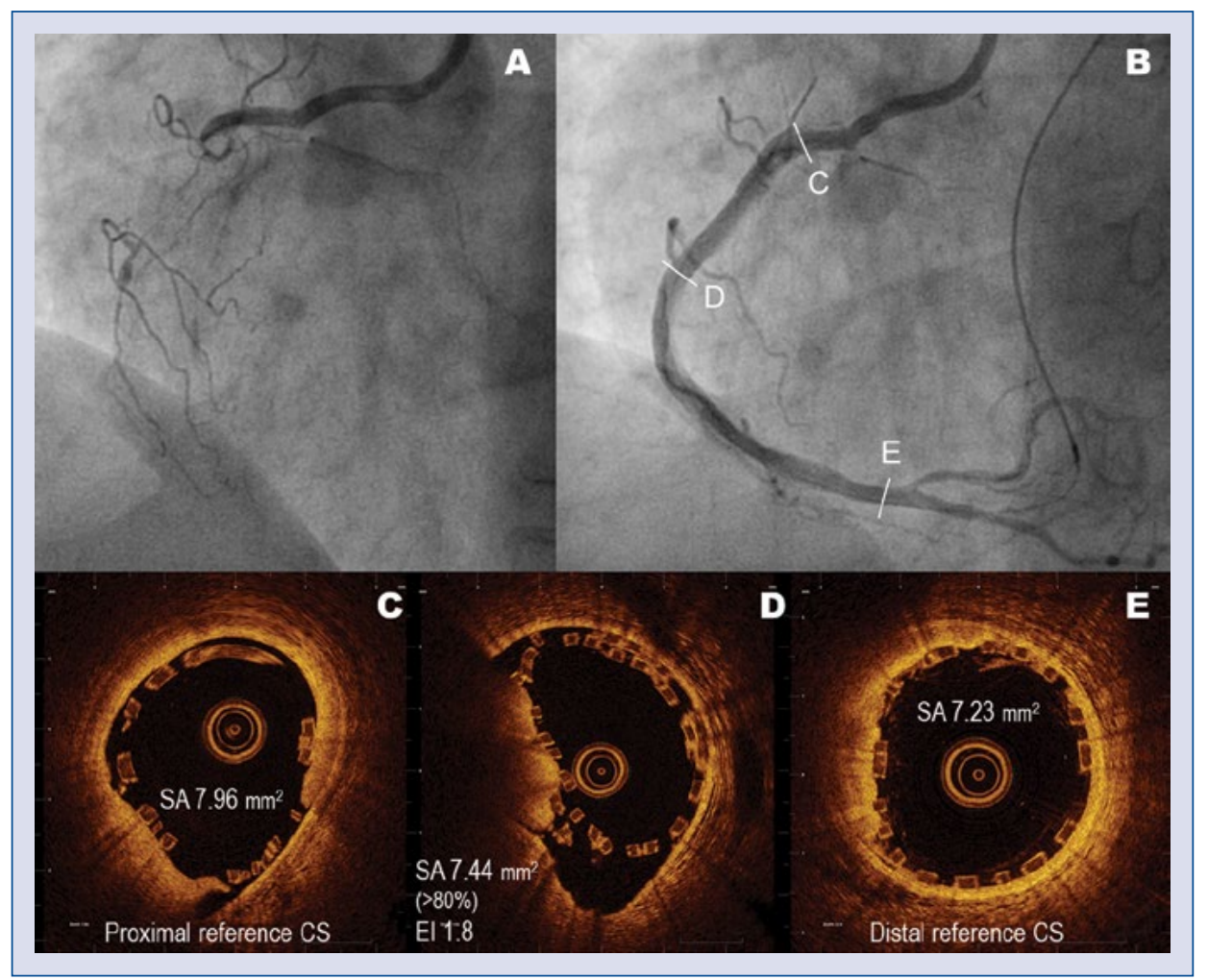

Figure 6. Case of persistent underexpansion due to ellipticity index (EI) > 1.6, notwithstanding repeat postdilation with non-compliant-balloons, in a segment of subintimal scaffolding for treatment of a chronic total occlusion; $\mathbf{A}$. Chronic total occlusion in the proximal right coronary artery; B. Final angiographic result after implantation of 3 DESOLVE scaffolds; C. Proximal reference cross-section (CS); D. Cross-section with minimal scaffold area (mm², thus not meeting the area criteria for underexpansion, but with $\mathrm{EI} \geq 1.6$ ); $\mathrm{E}$. Distal reference CS.

from $79.4 \%$ to $88.2 \%$ after post-dilating and this improvement was mainly due to the correction of ISA. The effect of postdilation on BRS underexpansion was marginal: only 1 case could be properly expanded.

\section{Limitations of the study}

The current study is a descriptive, singlecenter pilot study with a modest sample size. Although procedural and clinical results are promising, they must be cautiously interpreted due to the limited sample size. Furthermore, the hereby reported follow-up examines the early and late phases only, when scaffold thrombosis could be efficiently prevented, but remains uncertain whether OCT-guidance would have had any impact on very late scaffold thrombosis, which may to depend on different mechanisms such as mechanical instability or repeated stress provoked by dismantling [14]. Confirmation of these findings in a larger clinical study, making comparisons to current angiographic standards is required. Like- wise, reproducibility of the proposed methodology at different centres with different operators needs to be evaluated to estimate generalizability of the strategy.

Notwithstanding positive clinical outcomes of the study, despite one severe procedural complication (coronary rupture), which occurred in 1 patient. Coronary ruptures of unclear mechanism have been reported by other operators after BRS implantation [44]. In the present case an undisputed cause for the rupture could be elucidated after a careful review, although it could be most likely attributed to aggressive plaque preparation, as suggested by distal coronary haematoma visible in angiography and OCT after sealing the perforation. Nonetheless, the site of rupture was at the distal edge of a predilating compliant balloon, hence it was far from the point of cutting-balloon inflation. Although this complication did not finally result in a clinically adverse outcome, it was potentially life-threatening and should invite critical appraisals of the protocol. 
Table 4. Procedural and clinical outcomes.

\begin{tabular}{|c|c|}
\hline Parameters & $\begin{array}{l}\text { Protocol-compliant } \\
\text { patients }(n=28)\end{array}$ \\
\hline \multicolumn{2}{|l|}{ Peri-procedural outcomes } \\
\hline Death & $0(0.0 \%)$ \\
\hline Non-fatal MI & $1(3.6 \%)$ \\
\hline Stroke & $0(0.0 \%)$ \\
\hline Procedural success & $27(96.4 \%)$ \\
\hline \multicolumn{2}{|l|}{ In-hospital outcomes } \\
\hline Death & $0(0.0 \%)$ \\
\hline Non-fatal MI: & $1(3.6 \%)$ \\
\hline TV-related & $1(3.6 \%)$ \\
\hline Any revascularisation: & $0(0.0 \%)$ \\
\hline TLR & $0(0.0 \%)$ \\
\hline TVR & $0(0.0 \%)$ \\
\hline Stroke & $0(0.0 \%)$ \\
\hline $\begin{array}{l}\text { Patient-oriented endpoint } \\
\text { (death, MI, any } \\
\text { revascularization) }\end{array}$ & $1(3.6 \%)$ \\
\hline $\begin{array}{l}\text { MACCE (death, MI, any } \\
\text { revascularization, stroke) }\end{array}$ & $1(3.6 \%)$ \\
\hline $\begin{array}{l}\text { TVF (cardiac death, } \\
\text { TV-related MI, TVR) }\end{array}$ & $1(3.6 \%)$ \\
\hline \multicolumn{2}{|l|}{ 12-months follow-up } \\
\hline \multicolumn{2}{|l|}{ Death: } \\
\hline Cardiac & $0(0.0 \%)$ \\
\hline All-cause & $1(3.6 \%)$ \\
\hline Non-fatal MI & $2(7.1 \%)$ \\
\hline TV-related & $1(3.6 \%)$ \\
\hline Any revascularization: & $1(3.6 \%)$ \\
\hline TLR & $0(0.0 \%)$ \\
\hline TVR & $0(0.0 \%)$ \\
\hline Stroke & $0(0.0 \%)$ \\
\hline $\begin{array}{l}\text { Patient-oriented endpoint } \\
\text { (death, MI, any } \\
\text { revascularization) }\end{array}$ & $3(10.7 \%)$ \\
\hline $\begin{array}{l}\text { MACCE (death, MI, any } \\
\text { revascularization, stroke) }\end{array}$ & $3(10.7 \%)$ \\
\hline $\begin{array}{l}\text { TVF (cardiac death, } \\
\text { TV-related MI, TVR) }\end{array}$ & $1(3.6 \%)$ \\
\hline $\begin{array}{l}\text { Definite/probable/possible } \\
\text { scaffold thrombosis }\end{array}$ & $0(0.0 \%)$ \\
\hline
\end{tabular}

Data presented as number (percentage). MACCE — major adverse cardiac and cerebrovascular event; $\mathrm{MI}$ - myocardial infarction; TV - target vessel; TLR - target lesion revascularization; TVF target vessel failure; TVR - target vessel revascularization

\section{Conclusions}

Optical coherence tomography-guided BRS implantation was feasible in $90.2 \%$ of de novo lesions and resulted in optimal expansion and apposi- tion of scaffolds, correlating with $3.6 \%$ incidence of target vessel failure and $0 \%$ of early plus late scaffold thrombosis at $12^{\text {th }}$ month of follow-up, probably due to a better selection of lesions amenable for BRS treatment and to the possibility of tailoring intervention to predominant type of plaque. These encouraging pilot clinical results need to be confirmed in larger clinical studies.

\section{Conflict of interest: None declared}

\section{References}

1. Wykrzykowska JJ, Onuma Y, Serruys PW. Vascular restoration therapy: the fourth revolution in interventional cardiology and the ultimate "rosy" prophecy. EuroIntervention. 2009; 5 Suppl F: F7-F8, doi: 10.4244/EIJV5IFA1, indexed in Pubmed: 22100680.

2. Kereiakes DJ, Onuma Y, Serruys PW, et al. Bioresorbable Vascular Scaffolds for Coronary Revascularization. Circulation. 2016; 134(2): 168-182, doi: 10.1161/CIRCULATIONAHA.116.021539, indexed in Pubmed: 27400899.

3. Ormiston JA, Serruys PW, Regar E, et al. A bioabsorbable everolimus-eluting coronary stent system for patients with single de-novo coronary artery lesions (ABSORB): a prospective open-label trial. Lancet. 2008; 371(9616): 899-907, doi: 10.1016/ S0140-6736(08)60415-8, indexed in Pubmed: 18342684.

4. Serruys PW, Ormiston JA, Onuma Y, et al. A bioabsorbable everolimus-eluting coronary stent system (ABSORB): 2-year outcomes and results from multiple imaging methods. Lancet. 2009; 373(9667): 897-910, doi: 10.1016/S0140-6736(09)60325-1, indexed in Pubmed: 19286089.

5. Onuma Y, Serruys PW, Ormiston JA, et al. Three-year results of clinical follow-up after a bioresorbable everolimus-eluting scaffold in patients with de novo coronary artery disease: the ABSORB trial. EuroIntervention. 2010; 6(4): 447-453, doi: 10.4244/ EIJ30V6I4A76, indexed in Pubmed: 20884431.

6. Dudek D, Onuma Y, Ormiston JA, et al. Four-year clinical follow-up of the ABSORB everolimus-eluting bioresorbable vascular scaffold in patients with de novo coronary artery disease: the ABSORB trial. EuroIntervention. 2012; 7(9): 1060-1061, doi: 10.4244/EIJV7I9A168, indexed in Pubmed: 21959320.

7. Serruys PW, Onuma Y, Ormiston JA, et al. Evaluation of the second generation of a bioresorbable everolimus drug-eluting vascular scaffold for treatment of de novo coronary artery stenosis: six-month clinical and imaging outcomes. Circulation. 2010; 122(22): 2301-2312, doi: 10.1161/CIRCULATIONAHA.110.970772, indexed in Pubmed: 21098436.

8. Serruys PW, Onuma Y, Dudek D, et al. Evaluation of the second generation of a bioresorbable everolimus-eluting vascular scaffold for the treatment of de novo coronary artery stenosis: 12-month clinical and imaging outcomes. J Am Coll Cardiol. 2011; 58(15): 1578-1588, doi: 10.1016/j.jacc.2011.05.050, indexed in Pubmed: 21958884.

9. Serruys PW, Ormiston J, van Geuns RJ, et al. A polylactide bioresorbable scaffold eluting everolimus for treatment of coronary stenosis: 5-year follow-up. J Am Coll Cardiol. 2016; 67(7): 766-776, doi: 10.1016/j.jacc.2015.11.060, indexed in Pubmed: 26892411.

10. Capodanno D, Gori T, Nef H, et al. Percutaneous coronary intervention with everolimus-eluting bioresorbable vascular scaffolds 
in routine clinical practice: early and midterm outcomes from the European multicentre GHOST-EU registry. EuroIntervention. 2015; 10(10): 1144-1153, doi: 10.4244/EIJY14M07_11, indexed in Pubmed: 25042421.

11. Puricel S, Cuculi F, Weissner M, et al. Bioresorbable coronary scaffold thrombosis: multicenter comprehensive analysis of clinical presentation, mechanisms, and predictors. J Am Coll Cardiol. 2016; 67(8): 921-931, doi: 10.1016/j.jacc.2015.12.019, indexed in Pubmed: 26916481.

12. Serruys PW, Chevalier B, Sotomi Y, et al. Comparison of an everolimus-eluting bioresorbable scaffold with an everolimuseluting metallic stent for the treatment of coronary artery stenosis (ABSORB II): a 3 year, randomised, controlled, single-blind, multicentre clinical trial. Lancet. 2016; 388(10059): 2479-2491, doi: 10.1016/S0140-6736(16)32050-5, indexed in Pubmed: 27806897.

13. Wykrzykowska JJ, Kraak RP, Hofma SH, et al. AIDA Investigators. Bioresorbable Scaffolds versus Metallic Stents in Routine PCI. N Engl J Med. 2017; 376(24): 2319-2328, doi: 10.1056/ NEJMoa1614954, indexed in Pubmed: 28402237.

14. Räber L, Brugaletta S, Yamaji K, et al. Very late scaffold thrombosis: intracoronary imaging and histopathological and Spectroscopic findings. J Am Coll Cardiol. 2015; 66(17): 1901-1914, doi: 10.1016/j.jacc.2015.08.853, indexed in Pubmed: 26493663.

15. Muramatsu T, Onuma Y, García-García HM, et al. Incidence and short-term clinical outcomes of small side branch occlusion after implantation of an everolimus-eluting bioresorbable vascular scaffold: an interim report of 435 patients in the ABSORB-EXTEND single-arm trial in comparison with an everolimus-eluting metallic stent in the SPIRIT first and II trials. JACC Cardiovasc Interv. 2013; 6(3): 247-257, doi: 10.1016/j.jcin.2012.10.013, indexed in Pubmed: 23517836.

16. Mangiameli A, Ohno Y, Attizzani GF, et al. Neoatherosclerosis as the cause of late failure of a bioresorbable vascular scaffold. JACC Cardiovasc Interv. 2015; 8(4): 633-634, doi: 10.1016/j. jcin.2014.11.014, indexed in Pubmed: 25819179.

17. Bastante T, Rivero F, Benedicto A, et al. Recurrent neoatherosclerosis after bioresorbable vascular scaffold treatment of instent restenosis. JACC Cardiovasc Interv. 2015; 8(9): 1264-1265, doi: 10.1016/j.jcin.2015.03.031, indexed in Pubmed: 26292592.

18. Gutiérrez-Chico JL, Regar E, Nüesch E, et al. Delayed coverage in malapposed and side-branch struts with respect to well-apposed struts in drug-eluting stents: in vivo assessment with optical coherence tomography. Circulation. 2011; 124(5): 612-623, doi: 10.1161/CIRCULATIONAHA.110.014514, indexed in Pubmed: 21768536.

19. Gutiérrez-Chico JL, Wykrzykowska J, Nüesch E, et al. Vascular tissue reaction to acute malapposition in human coronary arteries: sequential assessment with optical coherence tomography. Circ Cardiovasc Interv. 2012; 5(1): 20-9, S1, doi: 10.1161/CIRCINTERVENTIONS.111.965301, indexed in Pubmed: 22319063.

20. Foin N, Gutiérrez-Chico JL, Nakatani S, et al. Incomplete stent apposition causes high shear flow disturbances and delay in neointimal coverage as a function of strut to wall detachment distance: implications for the management of incomplete stent apposition. Circ Cardiovasc Interv. 2014; 7(2): 180-189, doi: 10.1161/CIRCINTERVENTIONS.113.000931, indexed in Pubmed: 24642998.

21. Ormiston JA, De Vroey F, Serruys PW, et al. Bioresorbable polymeric vascular scaffolds: a cautionary tale. Circ Cardiovasc
Interv. 2011; 4(5): 535-538, doi: 10.1161/CIRCINTERVENTIONS.111.963710, indexed in Pubmed: 22010192.

22. Onuma Y, Serruys P, Muramatsu T, et al. Incidence and imaging outcomes of acute scaffold disruption and late structural discontinuity after implantation of the absorb everolimus-eluting fully bioresorbable vascular scaffold. JACC: Cardiovascular Interventions. 2014; 7(12): 1400-1411, doi: 10.1016/j.jcin.2014.06.016.

23. Prati F, Kodama T, Romagnoli E, et al. Suboptimal stent deployment is associated with subacute stent thrombosis: optical coherence tomography insights from a multicenter matched study. From the CLI Foundation investigators: the CLI-THRO study. Am Heart J. 2015; 169(2): 249-256, doi: 10.1016/j. ahj.2014.11.012, indexed in Pubmed: 25641534.

24. Rivero F, Benedicto A, Bastante T, et al. Subacute thrombosis of a bioresorbable vascular scaffold implanted for recurrent in-stent restenosis. EuroIntervention. 2015; 11(7): 780, doi: 10.4244/EIJY14M10_07, indexed in Pubmed: 25308302.

25. Ruiz-Salmerón RJ, Pereira S, de Araujo D. Bioresorbable vascular scaffold collapse causes subacute thrombosis. J Invasive Cardiol. 2014; 26(7): E98-E99, indexed in Pubmed: 24993999.

26. Karanasos A, Van Mieghem N, van Ditzhuijzen N, et al. Angiographic and optical coherence tomography insights into bioresorbable scaffold thrombosis: single-center experience. Circ Cardiovasc Interv. 2015; 8(5), doi: 10.1161/CIRCINTERVENTIONS.114.002369, indexed in Pubmed: 25969547.

27. Ormiston JA, Webber B, Ubod B, et al. Absorb everolimus-eluting bioresorbable scaffolds in coronary bifurcations: a bench study of deployment, side branch dilatation and post-dilatation strategies. EuroIntervention. 2015; 10(10): 1169-1177, doi: 10.4244/ EIJY14M05_08, indexed in Pubmed: 24835848.

28. Ormiston JA, Webber B, Ubod B, et al. An independent bench comparison of two bioresorbable drug-eluting coronary scaffolds (Absorb and DESolve) with a durable metallic drug-eluting stent (ML8/Xpedition). EuroIntervention. 2015; 11(1): 60-67, doi: 10.4244/EIJY15M02_03, indexed in Pubmed: 25680225.

29. Foin N, Lee R, Mattesini A, et al. Bioabsorbable vascular scaffold overexpansion: insights from in vitro post-expansion experiments. EuroIntervention. 2016; 11(12): 1389-1399, doi: 10.4244/ EIJY15M07_02, indexed in Pubmed: 26151954.

30. Farooq V, Gomez-Lara J, Brugaletta S, et al. Proximal and distal maximal luminal diameters as a guide to appropriate deployment of the ABSORB everolimus-eluting bioresorbable vascular scaffold: a sub-study of the ABSORB Cohort B and the on-going ABSORB EXTEND Single Arm Study. Catheter Cardiovasc Interv. 2012; 79(6): 880-888, doi: 10.1002/ccd.23177, indexed in Pubmed: 22514149 .

31. Windecker S, Kolh P, Alfonso P, et al. 2014 ESC/EACTS Guidelines on myocardial revascularization: The Task Force on Myocardial Revascularization of the European Society of Cardiology (ESC) and the European Association for Cardio-Thoracic Surgery (EACTS)Developed with the special contribution of the European Association of Percutaneous Cardiovascular Interventions (EAPCI). . Eur Heart J. 2014; 35(37): 2541-2619, doi: 10.1093/ eurheartj/ehu278.

32. Gutiérrez-Chico JL, Radu MD, Diletti R, et al. Spatial distribution and temporal evolution of scattering centers by optical coherence tomography in the poly(L-lactide) backbone of a bioresorbable vascular scaffold. Circ J. 2012; 76(2): 342-350, indexed in Pubmed: 22104034.

33. Sheehy A, Gutiérrez-Chico JL, Diletti R, et al. In vivo characterisation of bioresorbable vascular scaffold strut interfaces using optical coherence tomography with Gaussian line spread 
function analysis. EuroIntervention. 2012; 7(10): 1227-1235, doi: 10.4244/EIJV7I10A195, indexed in Pubmed: 22222916.

34. Oberhauser JP, Hossainy S, Rapoza RJ. Design principles and performance of bioresorbable polymeric vascular scaffolds. EuroIntervention. 2009; 5 Suppl F: F15-F22, doi: 10.4244/EIJV5IFA3, indexed in Pubmed: 22100671.

35. Schmidt W, Behrens P, Brandt-Wunderlich C, et al. In vitro performance investigation of bioresorbable scaffolds - Standard tests for vascular stents and beyond. Cardiovasc Revasc Med. 2016; 17(6): 375-383, doi: 10.1016/j.carrev.2016.05.001, indexed in Pubmed: 27266902.

36. Fajadet J, Haude M, Joner M, et al. Magmaris preliminary recommendation upon commercial launch: a consensus from the expert panel on 14 April 2016. EuroIntervention. 2016; 12(7): 828-833, doi: 10.4244/EIJV12I7A137, indexed in Pubmed: 27639734.

37. Gibson CM, de Lemos JA, Murphy SA, et al. TIMI Study Group. Combination therapy with abciximab reduces angiographically evident thrombus in acute myocardial infarction: a TIMI 14 substudy. Circulation. 2001; 103(21): 2550-2554, indexed in Pubmed: 11382722.

38. Prati F, Cera M, Ramazzotti V, et al. Safety and feasibility of a new non-occlusive technique for facilitated intracoronary optical coherence tomography (OCT) acquisition in various clinical and anatomical scenarios. EuroIntervention. 2007; 3(3): 365-370, indexed in Pubmed: 19737719.
39. Collet C, Suwannasom P, Almeida M, et al. Coronary bioresorbable vascular scaffold radiopaque marker embolization. Eur Heart J. 2017; 38(21): 1690, doi: 10.1093/eurheartj/ehx038, indexed in Pubmed: 28177449.

40. Gutiérrez-Chico JL, Alegría-Barrero E, Teijeiro-Mestre R, et al. Optical coherence tomography: from research to practice. Eur Heart J Cardiovasc Imaging. 2012; 13(5): 370-384, doi: 10.1093/ ehjci/jes025, indexed in Pubmed: 22330231.

41. Cutlip DE, Windecker S, Mehran R, et al. Academic Research Consortium. Clinical end points in coronary stent trials: a case for standardized definitions. Circulation. 2007; 115(17): 2344-2351, doi: 10.1161/ CIRCULATIONAHA.106.685313, indexed in Pubmed: 17470709

42. Cortés C, Jaguszewski M, Amat-Santos IJ, et al. Bas-relief in three dimensional optical coherence tomography: a novel sign after subintimal scaffolding in a chronic total occlusion. Eur Heart J. 2017; 38(31): 2445, doi: 10.1093/eurheartj/ehx126, indexed in Pubmed: 28329344.

43. Fabris E, Caiazzo G, Kilic ID, et al. Is high pressure postdilation safe in bioresorbable vascular scaffolds? Optical coherence tomography observations after noncompliant balloons inflated at more than 24 atmospheres. Catheter Cardiovasc Interv. 2016; 87(5): 839-846, doi: 10.1002/ccd.26222, indexed in Pubmed: 26370357.

44. Kawamoto H, Jabbour RJ, Tanaka A, et al. Contained coronary rupture following bioresorbable scaffold implantation in a small vessel. Int J Cardiol. 2016; 209: 24-25, doi: 10.1016/j. ijcard.2016.02.026, indexed in Pubmed: 26878469. 